\title{
A Hybrid Tabu Search Algorithm for a Real-World Open Vehicle Routing Problem Involving Fuel Consumption Constraints
}

\author{
Yunyun Niu $\mathbb{D}^{1},{ }^{1}$ Zehua Yang $\mathbb{D}^{1},{ }^{1}$ Ping Chen, ${ }^{2}$ and Jianhua Xiao $\mathbb{D D}^{3}$ \\ ${ }^{1}$ School of Information Engineering, China University of Geosciences, Beijing, Beijing 100083, China \\ ${ }^{2}$ School of Business, Nankai University, Tianjin 300071, China \\ ${ }^{3}$ The Research Center of Logistics, Nankai University, Tianjin 300071, China
}

Correspondence should be addressed to Jianhua Xiao; jhxiao2008@163.com

Received 4 October 2017; Accepted 1 February 2018; Published 28 February 2018

Academic Editor: Danilo Comminiello

Copyright (C) 2018 Yunyun Niu et al. This is an open access article distributed under the Creative Commons Attribution License, which permits unrestricted use, distribution, and reproduction in any medium, provided the original work is properly cited.

\begin{abstract}
Outsourcing logistics operation to third-party logistics has attracted more attention in the past several years. However, very few papers analyzed fuel consumption model in the context of outsourcing logistics. This problem involves more complexity than traditional open vehicle routing problem (OVRP), because the calculation of fuel emissions depends on many factors, such as the speed of vehicles, the road angle, the total load, the engine friction, and the engine displacement. Our paper proposed a green open vehicle routing problem (GOVRP) model with fuel consumption constraints for outsourcing logistics operations. Moreover, a hybrid tabu search algorithm was presented to deal with this problem. Experiments were conducted on instances based on realistic road data of Beijing, China, considering that outsourcing logistics plays an increasingly important role in China's freight transportation. Open routes were compared with closed routes through statistical analysis of the cost components. Compared with closed routes, open routes reduce the total cost by $18.5 \%$ with the fuel emissions cost down by nearly $29.1 \%$ and the diver cost down by $13.8 \%$. The effect of different vehicle types was also studied. Over all the 60 - and 120 -node instances, the mean total cost by using the light-duty vehicles is the lowest.
\end{abstract}

\section{Introduction}

Many developing countries are confronted with two problems: how to reduce economic costs and how to develop an environment-friendly society. As a result, researchers as well as companies with integrated logistics expertise tend to adopt new transportation models to counter these problems. Outsourcing logistics operations to the third-party logistics would reduce costs through better resource utilization and operations efficiency in freight transportation. It plays an increasingly important role in freight transportation. In that case, a company can hire vehicles from other companies to deliver its goods. Vehicles do not need return to the company as usual. They are usually described as the open vehicle routing problem (OVRP) [1]. Compared with the vehicle routing problem (VRP), routes in the OVRP model are open; see Figure 1.

The OVRP is NP-hard [2], so heuristics or metaheuristics methods are usually used to deal with it [3], such as the tabu search [4], the neighborhood-based search [5], particle swarm optimization [6], ant colony optimization [7], or evolutionary computing [8]. Hybrid metaheuristic algorithms were also designed to solve the OVRP [9]. Moreover, several variants of the OVRP were studied to model specific practical problems. OVRP with time windows (OVRPTW) was presented by [10] to model the delivery of multiproduct newspapers. The multidepot OVRP (MDOVRP) was first proposed by [11] to model the distribution of fresh meat. The open vehicle routing problem with decoupling points (OVRP-DP) was introduced in [12] to describe open routes performed by more than one carrier. The OVRP with uncertain demands was investigated to deal with nondeterministic customer demands and avoid unsatisfied demands or more extra operation cost [13]. However, among models studied in the past there are rare formulations considering the impact of fuel and carbon emission [14]. This paper is a contribution to this line of research. 


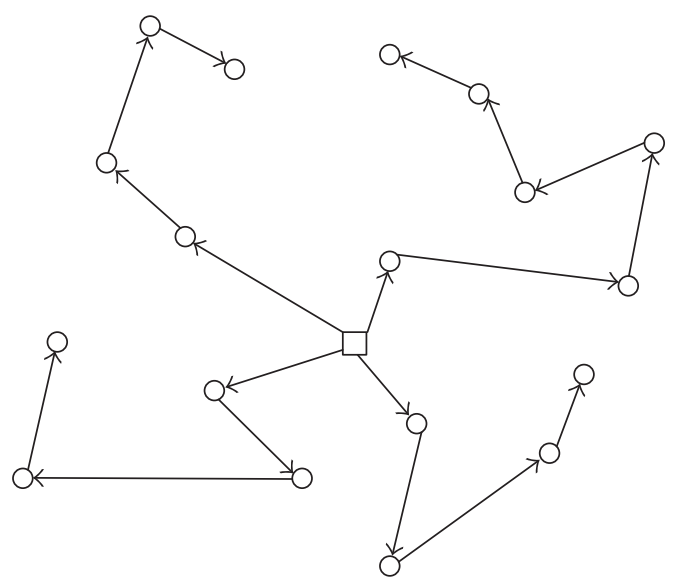

FIGURE 1: Open vehicle routing problem.

Carbon emissions along with freight transportation have hazardous impacts on the environment. How to minimize the fuel consumption becomes a hot topic. In [15], six fuel consumption models were compared with each other and several factors affecting carbon emissions in road transportation were analyzed. In [16], the pollution-routing problem (PRP) was introduced to evaluate the greenhouse gas emissions for the VRP. The total energy consumed on each road can be directly translated into greenhouse gas emissions with the PRP model. Reference [17] considered a heterogeneous vehicle fleet and extended the PRP model. Reference [18] presented a recent review of studies on green freight transportation problems.

This study has been motivated by a real problem in Beijing, China. As outsourcing logistics is playing a more and more important role in China's freight transportation, many companies hire vehicles from the third-party logistics. Vehicles do not need return to the depot after delivering products to customers. Each route departs from the depot and ends up with one of the customers. These companies are facing the problem of minimizing the total cost, including both fuel consumption cost and driver salaries.

The main contributions of our paper are summarized as follows. (1) Fuel consumption was analyzed in the context of the OVRP; and the green open vehicle routing problem (GOVRP) model was constructed. With the purpose of optimizing the fuel emissions cost in the model, we introduced the comprehensive modal emission model (CMEM) into the OVRP model and extend the OVRP with an objective that accounts for the amount of greenhouse emissions. (2) A hybrid tabu search algorithm involving several neighborhood search strategies was proposed to solve the GOVRP. A modified nearest neighborhood heuristic $(\mathrm{mNNH})$ was proposed to get the initial solution. It considered two factors load and distance when searching for a suitable initial solution. And then four neighborhood search operators were designed to produce the neighborhood of the current solution. (3) Computational experiments were conducted on instances derived from real geographical data of customers in Beijing, China. Both the effect of open routes and the effect of vehicle types were analyzed in respects of reducing total cost.

\section{Mathematical Model of the GOVRP}

The CMEM was developed by $[19,20]$ to calculate fuel emissions. In this section, we introduced the CMEM to the OVRP and formulated the mathematical model of the GOVRP. The fuel consumption $F^{h}$ (in liters) of vehicle type $h$ is given by [21]

$$
F^{h}=\lambda\left(\frac{k^{h} N^{h} V^{h} d}{v}+M^{h} \gamma^{h} \alpha d+\beta^{h} \gamma^{h} d v^{2}\right),
$$

where $\lambda=\xi / \kappa \psi, \gamma^{h}=1 / 1000 n_{t f} \eta, \alpha=\tau+g \sin \theta+g C_{r}^{h} \cos \theta$, and $\beta^{h}=0.5 C_{d} A^{h} \rho$ are constraints. $M^{h}$ is the total vehicle weight, $d$ is the distance, and $v$ is the vehicle speed. Three terms of $F^{h}$ are referred to as the engine module, the weight module, and the speed module, respectively. Notations and their default values are listed in the following two tables $[17,22]$. Common parameters of vehicles are listed in Table 1. Specific parameters for different vehicle types are listed in Table 2.

Let $N=\{0, \ldots, n\}$ be the set of customers and the depot, and let $A=\{(i, j): i, j \in N, i \neq j, j \neq 0\}$ be the set of arcs. Node 0 denotes the depot, so the customer set is $N_{0}=N \backslash$ $\{0\}$. $Q$ represents the capacity of vehicles. Variables $q_{i}$ means the demand of customer $i$. Variables $d_{i j}$ is the distance from node $i$ to node $j$. Variables $f_{i j}$ are the total amount of flow on arc $(i, j)$. The mathematical model of GOVRP is defined as follows:

$$
\begin{aligned}
\text { Minimize } & \sum_{(i, j) \in A} \lambda f_{c} k N V d_{i j} \sum_{r=1}^{R} \frac{z_{i j}^{r}}{v^{r}} \\
& +\sum_{(i, j) \in A} \lambda f_{c} \gamma \alpha_{i j} d_{i j}\left(w x_{i j}+f_{i j}\right) \\
& +\sum_{(i, j) \in A} \lambda f_{c} \beta \gamma d_{i j} \sum_{r=1}^{R}\left(v^{r}\right)^{2} z_{i j}^{r} \\
& +\sum_{j \in N_{0}} f_{d} s_{j} \\
& \sum_{j \in N_{0}} x_{0 j} \leq\left|N_{0}\right| \\
& \sum_{i \in N} x_{i j}=1, \quad \forall j \in N_{0} \\
& \sum_{j \in N} x_{i j} \leq 1, \quad \forall i \in N_{0} \\
& \sum_{j=1}^{R} z_{i j}^{r}=x_{i j}, \quad \forall(i, j) \in A \\
& \sum_{i=1}^{n} x_{i 0}=0 \\
& q_{j} x_{i j} \leq f_{i j} \leq\left(Q-\sum_{j \in N} f_{j i}=q_{i}, \quad \forall i \in N_{0}, \quad \forall(i, j) \in A\right. \\
&
\end{aligned}
$$


TABLE 1: Common parameters of vehicles.

\begin{tabular}{lcc}
\hline Notation & Description & Typical values \\
\hline$\xi$ & Fuel-to-air mass ratio & 1 \\
$g$ & Gravitational constant $\left(\mathrm{m} / \mathrm{s}^{2}\right)$ & 9.81 \\
$\rho$ & Air density $\left(\mathrm{kg} / \mathrm{m}^{3}\right)$ & 1.2041 \\
$C_{r}^{h}$ & Coefficient of rolling resistance & 0.01 \\
$\eta$ & Efficiency parameter for diesel engines & 0.45 \\
$f_{c}$ & Fuel and $\mathrm{CO}_{2}$ emissions cost $(\mathrm{RMB} / \mathrm{liter})$ & 12.0165 \\
$f_{d}$ & Driver wage $(\mathrm{RMB} / \mathrm{s})$ & 0.0111 \\
$\kappa$ & Heating value of a typical diesel fuel $(\mathrm{kj} / \mathrm{g})$ & 44 \\
$\psi$ & Conversion factor $(\mathrm{g} / \mathrm{s} \mathrm{to} \mathrm{L/s)}$ & 737 \\
$n_{t f}$ & Vehicle drive train efficiency & 0.45 \\
$v^{l}$ & Lower speed limit $(\mathrm{m} / \mathrm{s})$ & 0 \\
$v^{u}$ & Upper speed limit $(\mathrm{m} / \mathrm{s})$ & $27.8($ or $100 \mathrm{~km} / \mathrm{h})$ \\
$\tau$ & Acceleration $\left(\mathrm{m} / \mathrm{s}^{2}\right)$ & 0 \\
\hline
\end{tabular}

TABLE 2: Specific parameters for different vehicle types.

\begin{tabular}{lcccc}
\hline Notation & Description & Light duty & Medium duty & Heavy duty \\
\hline$w$ & Curb weight $(\mathrm{kg})$ & 3500 & 5500 & 14,000 \\
$Q$ & Maximum payload (kg) & 4000 & 12,500 & 26,000 \\
$k$ & Engine friction factor (kj/rev/liter) & 0.25 & 0.20 & 0.15 \\
$N$ & Engine speed (rev/s) & 38.34 & 36.67 & 30.0 \\
$V$ & Engine displacement (liter) & 4.5 & 6.9 & 0.7 \\
$C_{d}$ & Coefficient of aerodynamics drag & 0.6 & 8.0 & 0.9 \\
$A$ & Frontal surface area $\left(\mathrm{m}^{2}\right)$ & 7.0 & 8.0 & 10.0 \\
\hline
\end{tabular}

$$
\begin{aligned}
& x_{i j} \in\{0,1\}, \quad \forall(i, j) \in A \\
& z_{i j}^{r} \in\{0,1\}, \quad \forall(i, j) \in A \\
& f_{i j} \geq 0, \quad \forall(i, j) \in A .
\end{aligned}
$$

Formula (2)-(5) describes the objective of the GOVRP, where terms (2)-(4) calculate the total fuel consumption cost and term (5) measures the total driver wages. More specifically, $f_{c}$ is the fuel emissions cost per liter, and terms (2)-(4) calculate the fuel consumption costs induced by the engine module, the weight module, and the speed module, respectively. $s_{j}$ denotes the total time spent on a route where $j$ is the last served customer.

The binary variable $x_{i j}$ is equal to 1 if there is a vehicle traveling on arc $(i, j)$; otherwise, $x_{i j}$ is equal to 0 . Constraints (6)-(9) ensure that each customer is served by one vehicle and it is served only once. Vehicles do not return to the deport. If $i$ is the last served customer, constraint (8) can be written as $\sum_{j \in N} x_{i j}=0$. Otherwise, it can be written as $\sum_{j \in N} x_{i j}=1$. Constraints (10) and (11) define flows. The binary variable $z_{i j}^{r}$ is equal to 1 when a vehicle travels on $\operatorname{arc}(i, j)$ at speed $v^{r}$.

\section{Hybrid Tabu Search Algorithm for the GOVRP}

In this study, a hybrid tabu search algorithm including several neighborhood search strategies was designed to solve the

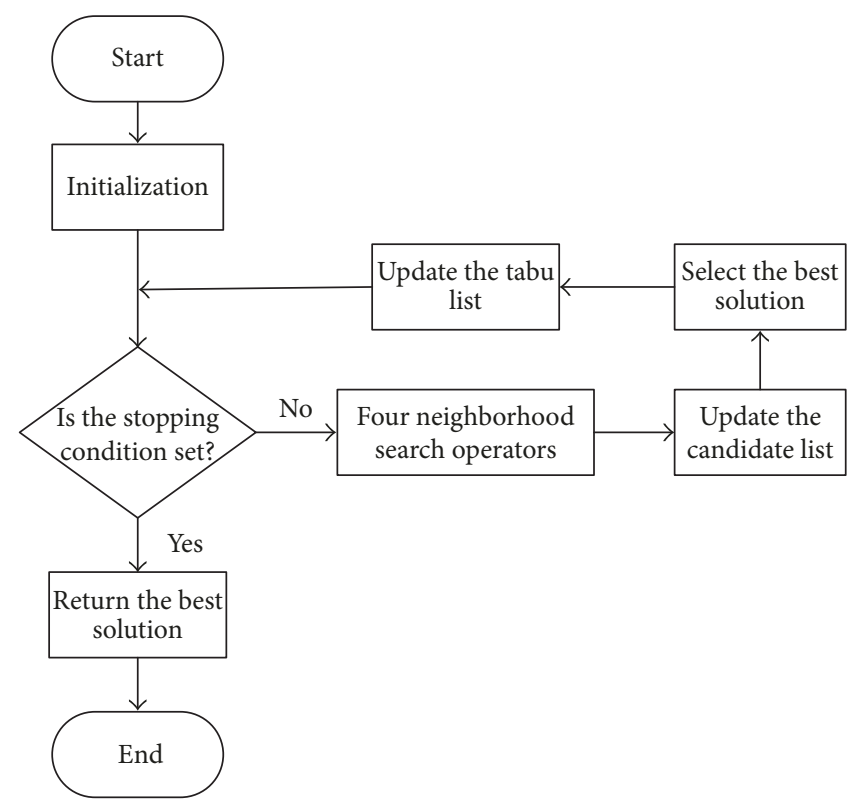

FIgURE 2: The flowchart of our algorithm.

GOVRP. The flowchart of this algorithm is shown in Figure 2. The detailed steps are listed as follows. 
Step 1. The initial setup includes initializing an empty tabu list and an empty candidate list, creating an initial solution, setting that initial solution as the best solution to date. A modified nearest neighborhood heuristic $(\mathrm{mNNH})$ is used to gain the initial solution. We will elaborate on it specifically in Section 3.1.

Step 2. If the stopping condition is satisfied, the search process stops, and the best solution is returned. Otherwise, it turns to Step 3.

Step 3. The solution space is explored each step from a current solution to the accepted solutions in the neighborhood by using four efficient neighborhood search algorithms. For each neighbor of the current solution $X_{\text {current }}$, the optimal speed on each arc of their routes is set by using the speed optimization algorithm according to [23].

Step 4. The neighboring solutions are checked for tabu elements. We search for the best solution and update the candidate list.

Step 5. If the best local candidate is better than the current best solution, update the current best solution. The local optimal solution is added to the tabu list. Then, turn to Step 2.

In this paper, the stopping condition is the maximum number of iterations $X_{\max }$. When our algorithm is iterated $X_{\max }$ times, the algorithm is terminated.

3.1. The Initial Solution. We use a modified nearest neighborhood heuristic ( $\mathrm{mNNH}$ ) to obtain an initial solution. The mNNH considers two factors load and distance when searching for a suitable initial solution. In a delivery system, a vehicle can reduce $q_{j}$ load after servicing customer $j$. We define

$$
\overline{\Delta f_{i j}}=\frac{q_{j}}{d_{i j}}, \quad i \in N, \quad j \in N_{\mathrm{ucs}},
$$

where $N_{\text {unc }}$ is defined as the unrouted customer set.

The mNNH builds routes one by one according to the following steps. The first route begins with an unrouted customer $r$, which can be calculated by the following equation:

$$
r=\underset{j \in N_{\mathrm{ucs}}}{\arg \max }\left\{\left|\overline{\Delta f_{0 j}}\right|\right\} \text {. }
$$

Then, we calculate $\overline{\Delta f_{i j}}$ between the current node $r$ and the other unrouted nodes. We choose the node with the greatest current $\overline{\Delta f_{i j}}$ value as the next node if the current route does not violate the vehicle capacity constraint. We update the current node $r$ and search for the next node in the same way. When no customer can be assigned to the route, a new route is started. When all the customers are already routed, the process stops. The algorithm is described as Algorithm 1.

3.2. The Neighborhood Search. The neighborhood of the current solution $X_{\text {current }}$ is first obtained by four neighborhood search operators. They cannot violate the capacity constraint
Input: $Q, q_{1}, \ldots, q_{n}$

Output: path
(1) Set $N_{\text {ucs }}=\{1, \ldots, n\}$, path $=\emptyset$, route $=\emptyset$
(2) while $N_{\text {ucs }} \neq \emptyset$ do
(3) Select a car
(4) Set $q \leftarrow Q$
(5) while $q-q_{j} \geq 0$ and $N_{\text {ucs }} \neq \emptyset$ do
(6) $\quad$ Set route $\leftarrow$ route $\cup\{r\}, q \leftarrow q-q_{j}, i \leftarrow j$
(7) $\quad N_{\text {ucs }} \leftarrow N_{\text {ucs }} /\{j\}, r \leftarrow \arg \max _{j \in N_{\text {ucs }}}\left\{\left|\overline{\Delta f_{i j}}\right|\right\}$
(8) Set path $\leftarrow$ path $\cup\{$ route $\}$, route $=\emptyset$
(9) return path

Algorithm 1: A modified nearest neighborhood heuristic.

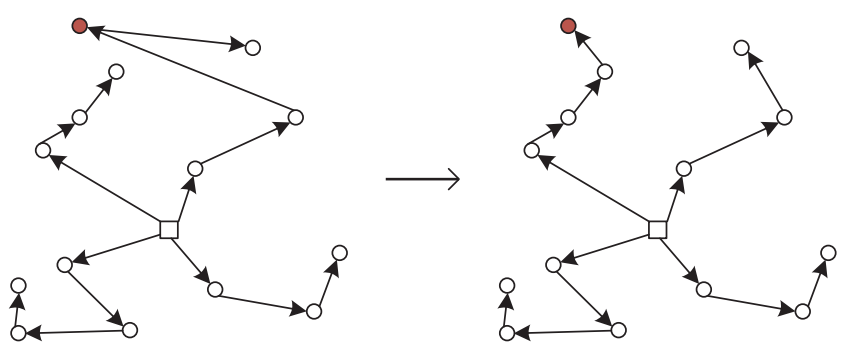

FIGURE 3: High-cost-node improvement operator.

TABLE 3: Algorithmic parameters values.

\begin{tabular}{lcc}
\hline Notation & Description & Typical values \\
\hline$X_{\max }$ & The maximum iteration number & 500 \\
$l_{t}$ & The length of Tabu list & 5 \\
$n_{1}$ & The number of RO in each step & 40 \\
$n_{2}$ & The number of HCNIO in each step & 5 \\
$l$ & The threshold in SRIO in each step & 3 \\
$p$ & Each route has a chance $p$ to be improved & $50 \%$ \\
\hline
\end{tabular}

during this process as before. Then, the optimal speed on each route is set according to [23].

(1) Random Operator ( $R O)$. It randomly selects one node from the solution and then randomly finds a possible position for it.

(2) High-Cost-Node Improvement Operator (HCNIO). As illustrated in Figure 3, the operator tries to reassign the high cost node $u^{*}$, because the total distance between its preceding customer and its following customer is the longest. Customer $u^{*}$ can be calculated as follows:

$$
u^{*}=\underset{u \in N}{\arg \max }\left\{d_{i u}+d_{u j}\right\},
$$

where $i$ is the preceding customer and $j$ is the following customer.

We compute the best possible position for node $u$ based on

$$
c\left(i^{*}, u, j^{*}\right)=\min [c(i, u, j)], \quad i, j \in N, i, j \neq N_{\mathrm{ucs}},
$$


TABLE 4: Cost components with Min TC.

\begin{tabular}{|c|c|c|c|c|c|c|}
\hline Instances & $\mathrm{TD}(\mathrm{m})$ & ET (s) & FEC (RMB) & $\mathrm{DC}(\mathrm{RMB})$ & TC (RMB) & CE (kg) \\
\hline BJ10_01 & 160000 & 1.52 & 641.04 & 1427.23 & 2068.27 & 123.77 \\
\hline BJ10_02 & 215400 & 1.21 & 860 & 1669.73 & 2529.73 & 166.04 \\
\hline BJ10_03 & 148200 & 1.43 & 598.82 & 1515.92 & 2114.74 & 115.61 \\
\hline BJ10_04 & 125700 & 1.32 & 512.95 & 1604.03 & 2116.98 & 99.03 \\
\hline BJ10_05 & 131600 & 1.39 & 527.49 & 1261.2 & 1788.69 & 101.84 \\
\hline BJ10_06 & 195700 & 1.28 & 800.49 & 1731.86 & 2532.35 & 154.55 \\
\hline BJ10_07 & 161200 & 1.51 & 629.72 & 1462.42 & 2092.15 & 121.58 \\
\hline BJ10_08 & 160500 & 1.46 & 643.06 & 1598.9 & 2241.96 & 124.15 \\
\hline BJ10_09 & 174300 & 1.29 & 708.01 & 1687.92 & 2395.93 & 136.69 \\
\hline BJ10_10 & 196900 & 1.32 & 794.67 & 1724.37 & 2519.03 & 153.42 \\
\hline BJ20_01 & 207050 & 1.78 & 847.71 & 2872.04 & 3719.75 & 163.67 \\
\hline BJ20_02 & 327870 & 1.89 & 1313.52 & 3411.58 & 4725.1 & 253.6 \\
\hline BJ20_03 & 214080 & 1.84 & 869.09 & 2954.44 & 3823.52 & 167.79 \\
\hline BJ20_04 & 255540 & 2.01 & 1012 & 2740.72 & 3752.73 & 195.39 \\
\hline BJ20_05 & 276920 & 1.88 & 1118.67 & 3355.55 & 4474.22 & 215.98 \\
\hline BJ20_06 & 289340 & 2.18 & 1144.59 & 3143.29 & 4287.89 & 220.98 \\
\hline BJ20_07 & 303290 & 1.74 & 1257.06 & 3656.81 & 4913.87 & 242.7 \\
\hline BJ20_08 & 272120 & 1.8 & 1118.93 & 2995.08 & 4114.01 & 216.03 \\
\hline BJ20_09 & 310000 & 1.93 & 1250.4 & 3023.52 & 4273.93 & 241.41 \\
\hline BJ20_10 & 273670 & 2.25 & 1086.06 & 2945.31 & 4031.37 & 209.68 \\
\hline BJ30_01 & 383030 & 2.52 & 1594.7 & 4489.58 & 6084.28 & 307.89 \\
\hline BJ30_02 & 413850 & 2.62 & 1653.91 & 4497.14 & 6151.06 & 319.32 \\
\hline BJ30_03 & 425160 & 2.27 & 1763.89 & 4932.42 & 6696.31 & 340.55 \\
\hline BJ30_04 & 422810 & 2.55 & 1735.05 & 4702.76 & 6437.8 & 334.98 \\
\hline BJ30_05 & 348850 & 2.82 & 1405.68 & 4627.92 & 6033.6 & 271.39 \\
\hline BJ30_06 & 459680 & 2.28 & 1907.54 & 4772.35 & 6679.89 & 368.28 \\
\hline BJ30_07 & 460900 & 2.51 & 1900.86 & 4826.39 & 6727.26 & 367 \\
\hline BJ30_08 & 423510 & 2.38 & 1742.33 & 4625.07 & 6367.4 & 336.39 \\
\hline BJ30_09 & 447060 & 2.35 & 1869.31 & 4768.26 & 6637.57 & 360.9 \\
\hline BJ30_10 & 281950 & 2.59 & 1141.17 & 4007.94 & 5149.11 & 220.32 \\
\hline
\end{tabular}

where $c(i, u, j)=d_{i u}+d_{u j}-d_{i j}$ is the cost for inserting node $u$ between customer $i$ and customer $j$ and $\overline{N_{\text {ucs }}} \subseteq N$ is the set of currently routed customers.

(3) Short-Route Improvement Operator (SRIO). The operator tries to combine or delete short routes by reassigning nodes belonging to those routes. A threshold $l$ is set to evaluate the length of a route. Only if the length of a route is shorter than the threshold $l$, the nodes in that route can be reassigned to other positions. The process can be illustrated in Figure 4 $(l=2)$. First, arcs in the shorter routes are deleted and then isolated nodes are assigned to other longer routes.

The best possible position for each unrouted node $u$ is also calculated by (19). By using (20), the most suitable customer is selected to be inserted in the route repeatedly. Unrouted nodes will be inserted to the current routes one by one.

$$
c^{\prime}\left(i^{*}, u^{*}, j^{*}\right)=\min \left[c\left(i^{*}, u, j^{*}\right)\right] .
$$

(4) Random-Route-Improvement Operator (RRIO). Each route in the current solution has a chance $p$ of being

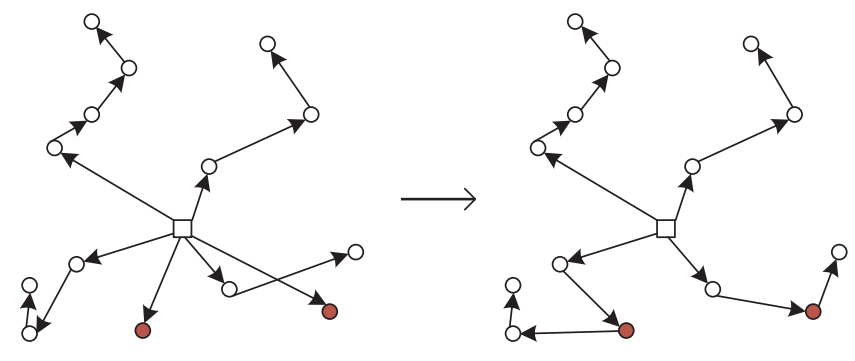

FIgURE 4: Short-route improvement operator.

destroyed. In that case, customers in that route should be reassigned. The best possible position for each node $u$ is calculated by (19). Then best customer to be inserted in the route is selected by using (20) repeatedly. All other unrouted customers will be inserted into the sequence one by one. As shown in Figure 5, two routes included in the dotted oval are destroyed; nodes in those routes are reassigned in possible positions. 
TABle 5: Cost components with Min FEC.

\begin{tabular}{|c|c|c|c|c|c|c|}
\hline Instances & $\mathrm{TD}(\mathrm{m})$ & ET (s) & FEC (RMB) & $\mathrm{DC}(\mathrm{RMB})$ & TC (RMB) & CE (kg) \\
\hline BJ10_01 & 160000 & 1.5 & 641.04 & 1427.23 & 2068.27 & 123.77 \\
\hline BJ10_02 & 215400 & 1.21 & 860 & 1669.73 & 2529.73 & 166.04 \\
\hline BJ10_03 & 148200 & 1.56 & 598.82 & 1515.92 & 2114.74 & 115.61 \\
\hline BJ10_04 & 126500 & 1.37 & 511.94 & 1607.36 & 2119.3 & 98.84 \\
\hline BJ10_05 & 132750 & 1.47 & 519.04 & 1265.98 & 1785.02 & 100.21 \\
\hline BJ10_06 & 195500 & 1.31 & 800.42 & 1731.02 & 2531.45 & 154.54 \\
\hline BJ10_07 & 161200 & 1.57 & 629.72 & 1462.42 & 2092.15 & 121.58 \\
\hline BJ10_08 & 161900 & 1.5 & 640.76 & 1604.72 & 2245.47 & 123.71 \\
\hline BJ10_09 & 174300 & 1.3 & 708.01 & 1687.92 & 2395.93 & 136.69 \\
\hline BJ10_10 & 196900 & 1.33 & 794.67 & 1724.37 & 2519.03 & 153.42 \\
\hline BJ20_01 & 207400 & 1.76 & 849.35 & 2873.49 & 3722.84 & 163.98 \\
\hline BJ20_02 & 328330 & 1.95 & 1309.37 & 3413.49 & 4722.86 & 252.8 \\
\hline BJ20_03 & 214900 & 1.97 & 872.32 & 2957.85 & 3830.17 & 168.42 \\
\hline BJ20_04 & 255610 & 2.18 & 1011.35 & 2741.01 & 3752.36 & 195.26 \\
\hline BJ20_05 & 277600 & 1.94 & 1120.84 & 3358.38 & 4479.21 & 216.4 \\
\hline BJ20_06 & 289930 & 2.25 & 1139.38 & 3145.75 & 4285.12 & 219.98 \\
\hline BJ20_07 & 304560 & 1.79 & 1262.84 & 3662.1 & 4924.93 & 243.81 \\
\hline BJ20_08 & 273430 & 1.88 & 1118.78 & 3000.53 & 4119.3 & 216 \\
\hline BJ20_09 & 309870 & 2.04 & 1247.69 & 3022.98 & 4270.67 & 240.89 \\
\hline BJ20_10 & 273750 & 2.25 & 1082.62 & 2945.65 & 4028.27 & 209.02 \\
\hline BJ30_01 & 382650 & 2.42 & 1589.04 & 4488 & 6077.04 & 306.79 \\
\hline BJ30_02 & 413260 & 2.74 & 1652.82 & 4494.69 & 6147.51 & 319.11 \\
\hline BJ30_03 & 421230 & 2.27 & 1747.69 & 4916.08 & 6663.77 & 337.42 \\
\hline BJ30_04 & 422830 & 2.43 & 1736.24 & 4702.84 & 6439.08 & 335.21 \\
\hline BJ30_05 & 352760 & 2.75 & 1417.82 & 4644.18 & 6062 & 273.74 \\
\hline BJ30_06 & 458930 & 2.19 & 1903.61 & 4769.23 & 6672.84 & 367.53 \\
\hline BJ30_07 & 458810 & 2.49 & 1884.59 & 4817.7 & 6702.3 & 363.86 \\
\hline BJ30_08 & 426290 & 2.32 & 1748.32 & 4636.63 & 6384.94 & 337.54 \\
\hline BJ30_09 & 452450 & 2.3 & 1890.76 & 4790.68 & 6681.44 & 365.05 \\
\hline BJ30_10 & 281430 & 2.6 & 1135.95 & 4005.78 & 5141.73 & 219.32 \\
\hline
\end{tabular}

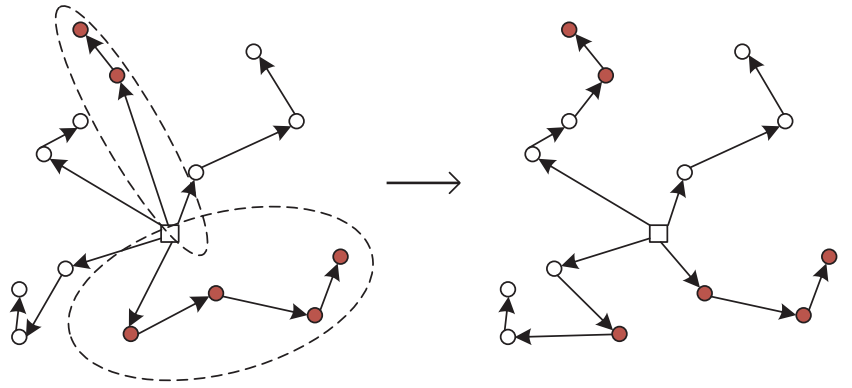

FIGURE 5: Random-route-improvement operator.

For each neighbor of the current solution $X_{\text {current }}$, the optimal speed on each route is set. According to [23], the optimal speed is

$$
v^{*}=\left(\frac{k N V}{2 \beta \gamma}+\frac{f_{d}}{2 \beta \gamma f_{c}}\right)^{1 / 3} .
$$

\section{Computational Analysis}

Experiments are run with data derived from real geographical distances of customers in Beijing. Three smaller classes with 10,20 , and 30 customers and four larger classes with 60, 80, 100 , and 120 customers are generated. Each class includes 10 instances. All the customers involved in this study are shown in Figure 6. The algorithmic parameters values are given in Table 3.

4.1. Effect of Objectives Min TD, Min FEC and Min DC. Three different objectives, Min TD (total distance), Min FEC (fuel emissions cost), and Min DC (driver cost), are used to minimize the total distance, fuel emissions cost, and driver cost, respectively. We obtained solutions with different cost components on each performance measure and analyzed the effect of different objectives. Experiments were conducted on 10-, 20-, and 30-node instances by using light-duty vehicles. The mean result of each instance collected over ten runs is reported; see Tables 4, 5, and 6. The columns display total distance (TD), execution time (ET), fuel emissions cost 
TABLE 6: Cost component with Min DC.

\begin{tabular}{|c|c|c|c|c|c|c|}
\hline Instances & $\mathrm{TD}(\mathrm{m})$ & $\mathrm{ET}(\mathrm{s})$ & FEC (RMB) & $\mathrm{DC}(\mathrm{RMB})$ & TC (RMB) & $\mathrm{CE}(\mathrm{kg})$ \\
\hline BJ10_01 & 159400 & 1.44 & 644.62 & 1424.74 & 2069.36 & 124.46 \\
\hline BJ10_02 & 215400 & 1.21 & 860 & 1669.73 & 2529.73 & 166.04 \\
\hline BJ10_03 & 148200 & 1.47 & 598.82 & 1515.92 & 2114.74 & 115.61 \\
\hline BJ10_04 & 125200 & 1.39 & 518.15 & 1601.95 & 2120.11 & 100.04 \\
\hline BJ10_05 & 131600 & 1.39 & 530.81 & 1261.2 & 1792 & 102.48 \\
\hline BJ10_06 & 195620 & 1.3 & 800.46 & 1731.52 & 2531.99 & 154.54 \\
\hline BJ10_07 & 161200 & 1.45 & 629.72 & 1462.42 & 2092.15 & 121.58 \\
\hline BJ10_08 & 160400 & 1.48 & 646.39 & 1598.48 & 2244.87 & 124.8 \\
\hline BJ10_09 & 174300 & 1.29 & 709.63 & 1687.92 & 2397.55 & 137.01 \\
\hline BJ10_10 & 196900 & 1.32 & 794.67 & 1724.37 & 2519.03 & 153.42 \\
\hline BJ20_01 & 206800 & 1.74 & 846.78 & 2871 & 3717.78 & 163.49 \\
\hline BJ20_02 & 329150 & 1.82 & 1327.82 & 3416.9 & 4744.72 & 256.36 \\
\hline BJ20_03 & 215720 & 1.81 & 879.27 & 2961.26 & 3840.53 & 169.76 \\
\hline BJ20_04 & 256490 & 1.99 & 1018.96 & 2744.67 & 3763.63 & 196.73 \\
\hline BJ20_05 & 280140 & 1.85 & 1134.25 & 3368.94 & 4503.19 & 218.99 \\
\hline BJ20_06 & 292080 & 2.1 & 1158.64 & 3154.69 & 4313.33 & 223.7 \\
\hline BJ20_07 & 303580 & 1.7 & 1259.44 & 3658.02 & 4917.46 & 243.16 \\
\hline BJ20_08 & 272670 & 1.78 & 1123.32 & 2997.37 & 4120.69 & 216.88 \\
\hline BJ20_09 & 310710 & 1.89 & 1254.84 & 3026.47 & 4281.31 & 242.27 \\
\hline BJ20_10 & 271600 & 2.18 & 1076.31 & 2936.7 & 4013.01 & 207.8 \\
\hline BJ30_01 & 381430 & 2.35 & 1589.86 & 4482.93 & 6072.79 & 306.95 \\
\hline BJ30_02 & 420450 & 2.47 & 1689.85 & 4524.59 & 6214.44 & 326.26 \\
\hline BJ30_03 & 427000 & 2.15 & 1773.68 & 4940.07 & 6713.75 & 342.44 \\
\hline BJ30_04 & 422740 & 2.35 & 1748.59 & 4702.47 & 6451.05 & 337.6 \\
\hline BJ30_05 & 352590 & 2.57 & 1423.67 & 4643.47 & 6067.14 & 274.87 \\
\hline BJ30_06 & 458020 & 2.17 & 1904.47 & 4765.45 & 6669.91 & 367.69 \\
\hline BJ30_07 & 459540 & 2.33 & 1897.68 & 4820.74 & 6718.42 & 366.38 \\
\hline BJ30_08 & 430970 & 2.21 & 1784.59 & 4656.09 & 6440.68 & 344.55 \\
\hline BJ30_09 & 445190 & 2.18 & 1864.22 & 4760.49 & 6624.71 & 359.92 \\
\hline BJ30_10 & 282940 & 2.44 & 1146.82 & 4012.06 & 5158.88 & 221.42 \\
\hline
\end{tabular}

TABLE 7: Cost components with different objectives.

\begin{tabular}{lccccccc}
\hline Instances & Objective & TD $(\mathrm{m})$ & ET $(\mathrm{s})$ & FEC (RMB) & DC (RMB) & TC (RMB) & CE (kg) \\
\hline \multirow{3}{*}{10 -node } & Min FEC & 167265 & 1.41 & 670.44 & 1569.67 & 2240.11 & 129.44 \\
& Min DC & 166822 & 1.37 & 673.33 & 1567.83 & 2241.15 & 130 \\
& Min TC & 166950 & 1.37 & 671.63 & 1568.36 & 2239.98 & 129.67 \\
\hline \multirow{3}{*}{20 -node } & Min FEC & 273538 & 2 & 1101.45 & 3112.12 & 4213.57 & 212.66 \\
& Min DC & 273894 & 1.89 & 1107.96 & 3113.6 & 4221.57 & 213.91 \\
& Min TC & 272988 & 1.93 & 1101.8 & 3109.83 & 4211.64 & 212.72 \\
\hline \multirow{3}{*}{30 -node } & Min FEC & 407064 & 2.45 & 1670.68 & 4626.58 & 6297.27 & 322.56 \\
& Min DC & 408087 & 2.32 & 1682.34 & 4630.84 & 6313.18 & 324.81 \\
& Min TC & 406680 & 2.49 & 1671.44 & 4624.98 & 6296.43 \\
\hline
\end{tabular}

(FEC), driver cost (DC), total cost (TC), and $\mathrm{CO}_{2}$ emissions (CE). Table 7 presents the average results of each class.

On average of 10-node class, the fuel emissions cost represents about $30 \%$ of the total cost. For 20 - and 30-node classes, the fuel emissions cost accounts for approximately $26 \%$ of the total cost. The carbon dioxide emissions increase with the number of customers. Only considering the driver cost in the objective leads to the most carbon dioxide emissions and the poorest total cost performance. Only considering the fuel emissions gets solutions with the lowest carbon dioxide emissions, but a higher total cost. Min TC as an objective yields the lowest total cost and the shortest total distance. 
TABLE 8: Cost components with closed routes.

\begin{tabular}{|c|c|c|c|c|c|c|}
\hline Instances & $\mathrm{TD}(\mathrm{m})$ & ET (s) & FEC (RMB) & $\mathrm{DC}(\mathrm{RMB})$ & TC (RMB) & $\mathrm{CE}(\mathrm{kg})$ \\
\hline BJ10_01 & 243300 & 1.35 & 973.42 & 1773.67 & 2747.08 & 187.94 \\
\hline BJ10_02 & 287900 & 1.23 & 1143.69 & 1971.25 & 3114.95 & 220.81 \\
\hline BJ10_03 & 233000 & 1.28 & 929.49 & 1868.6 & 2798.09 & 179.46 \\
\hline BJ10_04 & 193600 & 1.33 & 774.88 & 1886.42 & 2661.31 & 149.61 \\
\hline BJ10_05 & 190300 & 1.33 & 752.29 & 1505.32 & 2257.61 & 145.24 \\
\hline BJ10_06 & 306900 & 1.26 & 1216.62 & 2194.33 & 3410.94 & 234.89 \\
\hline BJ10_07 & 225500 & 1.19 & 914.3 & 1729.84 & 2644.14 & 176.52 \\
\hline BJ10_08 & 222400 & 1.33 & 883.91 & 1856.33 & 2740.24 & 170.65 \\
\hline BJ10_09 & 250200 & 1.29 & 1001.88 & 2003.58 & 3005.46 & 193.43 \\
\hline BJ10_10 & 285200 & 1.3 & 1133.7 & 2091.6 & 3225.3 & 218.88 \\
\hline BJ20_01 & 296880 & 1.75 & 1191.94 & 3245.63 & 4437.57 & 230.13 \\
\hline BJ20_02 & 432490 & 1.74 & 1733.98 & 3846.68 & 5580.66 & 334.78 \\
\hline BJ20_03 & 302470 & 1.93 & 1227.85 & 3322.04 & 4549.9 & 237.06 \\
\hline BJ20_04 & 350360 & 1.8 & 1408.36 & 3135.07 & 4543.43 & 271.91 \\
\hline BJ20_05 & 397350 & 1.75 & 1601.07 & 3856.4 & 5457.48 & 309.12 \\
\hline BJ20_06 & 404900 & 1.76 & 1627.02 & 3623.9 & 5250.92 & 314.13 \\
\hline BJ20_07 & 453910 & 1.79 & 1833.88 & 4283.23 & 6117.1 & 354.06 \\
\hline BJ20_08 & 377970 & 1.71 & 1526.82 & 3435.3 & 4962.12 & 294.78 \\
\hline BJ20_09 & 458430 & 1.79 & 1842.5 & 3640.83 & 5483.32 & 355.73 \\
\hline BJ20_10 & 380810 & 1.75 & 1533.55 & 3390.9 & 4924.45 & 296.08 \\
\hline BJ30_01 & 544130 & 2.53 & 2213.58 & 5159.58 & 7373.16 & 427.37 \\
\hline BJ30_02 & 602750 & 2.32 & 2434.58 & 5282.76 & 7717.34 & 470.04 \\
\hline BJ30_03 & 603420 & 2.25 & 2446.55 & 5673.79 & 8120.33 & 472.35 \\
\hline BJ30_04 & 574210 & 2.51 & 2328.31 & 5332.41 & 7660.72 & 449.52 \\
\hline BJ30_05 & 495510 & 2.46 & 2006.16 & 5237.86 & 7244.02 & 387.33 \\
\hline BJ30_06 & 636960 & 2.23 & 2584.8 & 5509.64 & 8094.44 & 499.04 \\
\hline BJ30_07 & 666890 & 2.43 & 2711.67 & 5683.09 & 8394.76 & 523.54 \\
\hline BJ30_08 & 588990 & 2.25 & 2385.82 & 5313.28 & 7699.1 & 460.63 \\
\hline BJ30_09 & 640480 & 2.22 & 2600.55 & 5572.68 & 8173.23 & 502.08 \\
\hline BJ30_10 & 394830 & 2.35 & 1599.07 & 4477.4 & 6076.47 & 308.73 \\
\hline
\end{tabular}

TABle 9: Cost components of different types of routes.

\begin{tabular}{lccccccc}
\hline Instances & Type of routes & TD $(\mathrm{m})$ & ET $(\mathrm{s})$ & FEC (RMB) & DC (RMB) & TC (RMB) & CE (kg) \\
\hline \multirow{2}{*}{10 -node } & Open & 166950 & 1.37 & 671.63 & 1568.36 & 2239.98 & 129.67 \\
& Closed & 243830 & 1.29 & 972.42 & 1888.09 & 2860.51 & 187.74 \\
\hline \multirow{2}{*}{20 -node } & Open & 272988 & 1.93 & 1101.8 & 3109.83 & 4211.64 & 212.72 \\
& Closed & 385557 & 1.78 & 1552.7 & 3578 & 5130.7 & 299.78 \\
\hline \multirow{2}{*}{30 -node } & Open & 406680 & 2.49 & 1671.44 & 4624.98 & 6296.43 & 322.7 \\
& Closed & 574817 & 2.36 & 2331.11 & 5324.25 & 7655.36 & 450.06 \\
\hline
\end{tabular}

4.2. Effect of Open Routes. In this subsection, we compared the total cost of open routes with that of closed routes. Experiments were also conducted on smaller instance classes by using light-duty vehicles. The mean results of closed routes collected over ten runs are listed in Table 8 . The average results of each class are reported in Table 9 to illustrate the effect of open routes. As shown in Table 9, open routes reduce the total cost by $18.5 \%$ with the fuel emissions cost down by nearly $29.1 \%$ and the diver cost down by $13.8 \%$ over all the instances.

4.3. Results for Larger Scale Instances. Experiments were also conducted on the four largest sets with the objective Min TC. The results collected over ten runs are reported in Table 10 by using light-duty vehicles. Notations BS, MS, WS, SD, and ET in columns represent the best solution, mean solution, 
TABLE 10: Computational results on larger scale instances.

\begin{tabular}{|c|c|c|c|c|c|}
\hline Instance & BS (RMB) & MS (RMB) & WS (RMB) & SD & ET (s) \\
\hline BJ60_01 & 11061.79 & 11254.42 & 11417.58 & 92.8 & 5.37 \\
\hline BJ60_02 & 10309.83 & 10393.21 & 10492.87 & 62.43 & 4.88 \\
\hline BJ60_03 & 11574.38 & 11790.03 & 11933.82 & 94.03 & 5.06 \\
\hline BJ60_04 & 12094.39 & 12212.64 & 12462.25 & 95.54 & 5.14 \\
\hline BJ60_05 & 11236.35 & 11320.09 & 11431.91 & 52.74 & 5.44 \\
\hline BJ60_06 & 11883.87 & 12040.48 & 12249.58 & 94.39 & 4.89 \\
\hline BJ60_07 & 12600.43 & 12745.03 & 12867.3 & 92.99 & 4.74 \\
\hline BJ60_08 & 11939.78 & 12060.86 & 12173.83 & 59.37 & 4.73 \\
\hline BJ60_09 & 11583.85 & 11727.88 & 11922.82 & 95.35 & 4.7 \\
\hline BJ60_10 & 12938.11 & 13121.2 & 13364.03 & 114.64 & 4.74 \\
\hline BJ80_01 & 14787.03 & 15036.55 & 15227.3 & 127.09 & 7.56 \\
\hline BJ80_02 & 14018.34 & 14220.78 & 14411.5 & 115.1 & 7.18 \\
\hline BJ80_03 & 14714.36 & 14964.93 & 15139.74 & 125.11 & 7.72 \\
\hline BJ80_04 & 13872.84 & 14065.74 & 14550.33 & 199.54 & 7.6 \\
\hline BJ80_05 & 14613.9 & 14788.56 & 15078.73 & 148.41 & 7.52 \\
\hline BJ80_06 & 16857.87 & 17148.49 & 17572.08 & 217.41 & 6.83 \\
\hline BJ80_07 & 15371.73 & 15632.16 & 15925.82 & 170.46 & 7.1 \\
\hline BJ80_08 & 14273.97 & 14484.07 & 14815.66 & 183.64 & 7.39 \\
\hline BJ80_09 & 14526.1 & 14743.32 & 14969.09 & 122.54 & 7.19 \\
\hline BJ80_10 & 14736.48 & 14942.92 & 15284.86 & 137.4 & 7.09 \\
\hline BJ100_01 & 19330.25 & 19617.94 & 20069.92 & 203.78 & 10.22 \\
\hline BJ100_02 & 20194.9 & 20701.34 & 21013.24 & 215.65 & 10.33 \\
\hline BJ100_03 & 20081.75 & 20286.97 & 20556.28 & 138.13 & 10.38 \\
\hline BJ100_04 & 18483.02 & 18689.28 & 18893.14 & 114.28 & 9.87 \\
\hline BJ100_05 & 18776.99 & 18983.5 & 19256.85 & 165.22 & 10.01 \\
\hline BJ100_06 & 17478.28 & 17764.38 & 18119.14 & 187.27 & 10.5 \\
\hline BJ100_07 & 18451.61 & 18775.37 & 18938.18 & 167.43 & 10.49 \\
\hline BJ100_08 & 18527.85 & 18762.86 & 18941.62 & 142.11 & 10.52 \\
\hline BJ100_09 & 17458.07 & 18048.33 & 18385.66 & 259.49 & 10.92 \\
\hline BJ100_10 & 20847 & 21099.27 & 21541.13 & 201.04 & 10 \\
\hline BJ120_01 & 21699.63 & 21954.27 & 22209.26 & 135.47 & 15.38 \\
\hline BJ120_02 & 22803.9 & 23135.32 & 23736.67 & 273.1 & 16.09 \\
\hline BJ120_03 & 22074.76 & 22569.41 & 22921.39 & 270.03 & 17.46 \\
\hline BJ120_04 & 22380.9 & 22560.26 & 22764.57 & 138.1 & 16.91 \\
\hline BJ120_05 & 22602.33 & 23308.71 & 23666.93 & 289.5 & 14.39 \\
\hline BJ120_06 & 22656.5 & 22975.25 & 23166.4 & 145.56 & 14.44 \\
\hline BJ120_07 & 21156.14 & 21711.99 & 22170.6 & 301.69 & 14.37 \\
\hline BJ120_08 & 20837.63 & 21069.17 & 21418.09 & 211.61 & 15.66 \\
\hline BJ120_09 & 21865.67 & 22195.93 & 22498.88 & 170.26 & 14.43 \\
\hline BJ120_10 & 22705.98 & 23054.33 & 23556.8 & 217.54 & 14.4 \\
\hline
\end{tabular}

the worst solution, standard deviation, and execution time, respectively. As shown in Table 10, the total cost increases with the number of customers.

4.4. Results with Different Vehicle Types. Experiments were conducted on the 60 - and 120-node instances by using different types of vehicles. In this subsection, the analysis focuses on which type of vehicle is the most suitable to minimize the total cost. The specific vehicle parameters are listed in Table 2. Results with different vehicle types are listed in Tables 11 and 12. Over all the instances in the two classes, the mean total cost by using the light-duty vehicles is the lowest. For 120-node instances, the mean total cost by using medium-duty vehicles increases by $4.8 \%$ and the result by using heavy-duty vehicles rises $23.6 \%$. For 60 -node instances, the mean total cost by using medium-duty vehicles increases by $7.7 \%$ and the value by using heavy-duty vehicles rises $28 \%$. 
TABLE 11: Computational results on the 60-node instances by using different vehicles.

\begin{tabular}{|c|c|c|c|c|c|c|}
\hline Type of vehicles & Instance & BS (RMB) & MS (RMB) & WS (RMB) & $\mathrm{SD}$ & $\mathrm{ET}(\mathrm{s})$ \\
\hline \multirow{10}{*}{ Only light duty } & BJ60_01 & 11061.79 & 11254.42 & 11417.58 & 92.8 & 5.37 \\
\hline & BJ60_02 & 10309.83 & 10393.21 & 10492.87 & 62.43 & 4.88 \\
\hline & BJ60_03 & 11574.38 & 11790.03 & 11933.82 & 94.03 & 5.06 \\
\hline & BJ60_04 & 12094.39 & 12212.64 & 12462.25 & 95.54 & 5.14 \\
\hline & BJ60_05 & 11236.35 & 11320.09 & 11431.91 & 52.74 & 5.44 \\
\hline & BJ60_06 & 11883.87 & 12040.48 & 12249.58 & 94.39 & 4.89 \\
\hline & BJ60_07 & 12600.43 & 12745.03 & 12867.3 & 92.99 & 4.74 \\
\hline & BJ60_08 & 11939.78 & 12060.86 & 12173.83 & 59.37 & 4.73 \\
\hline & BJ60_09 & 11583.85 & 11727.88 & 11922.82 & 95.35 & 4.7 \\
\hline & BJ60_10 & 12938.11 & 13121.2 & 13364.03 & 114.64 & 4.74 \\
\hline \multirow{10}{*}{ Only medium duty } & BJ60_01 & 12449.13 & 12616.53 & 12823.93 & 109.15 & 4.51 \\
\hline & BJ60_02 & 11328.74 & 11557.08 & 11780.96 & 125.14 & 4.19 \\
\hline & BJ60_03 & 12959.56 & 13222.5 & 13390.12 & 121.83 & 4.42 \\
\hline & BJ60_04 & 13411.61 & 13630.23 & 13870.71 & 147.61 & 4.4 \\
\hline & BJ60_05 & 12515.46 & 12678.95 & 12843.89 & 87.75 & 4.63 \\
\hline & BJ60_06 & 13037.75 & 13302.09 & 13413.95 & 100.83 & 4.29 \\
\hline & BJ60_07 & 12360.13 & 12425.3 & 12579.82 & 63.71 & 4.22 \\
\hline & BJ60_08 & 12942.29 & 13064.34 & 13360.38 & 121.13 & 4.49 \\
\hline & BJ60_09 & 12112.23 & 12348.68 & 12563.22 & 122.72 & 4.43 \\
\hline & BJ60_10 & 12832.96 & 12974.52 & 13105.98 & 88.75 & 4.03 \\
\hline \multirow{10}{*}{ Only heavy duty } & BJ60_01 & 14787.24 & 14995.59 & 15334.52 & 176.58 & 9.38 \\
\hline & BJ60_02 & 13418.08 & 13700.42 & 13911.3 & 143.14 & 7.91 \\
\hline & BJ60_03 & 15469.9 & 15746.78 & 16052.25 & 183.26 & 7.54 \\
\hline & BJ60_04 & 16164.2 & 16411.77 & 16729.41 & 172.11 & 7.78 \\
\hline & BJ60_05 & 14772.72 & 14937.7 & 15269.37 & 154.91 & 7.98 \\
\hline & BJ60_06 & 15893.22 & 16093.82 & 16272.75 & 133.43 & 7.41 \\
\hline & BJ60_07 & 14441.09 & 14606.23 & 14757.43 & 103.53 & 7.46 \\
\hline & BJ60_08 & 15088.73 & 15473.2 & 15638.19 & 152.24 & 7.61 \\
\hline & BJ60_09 & 14521.68 & 14811.72 & 14972.54 & 119.53 & 7.22 \\
\hline & BJ60_10 & 14961.08 & 15164.55 & 15321.96 & 109.22 & 6.88 \\
\hline
\end{tabular}

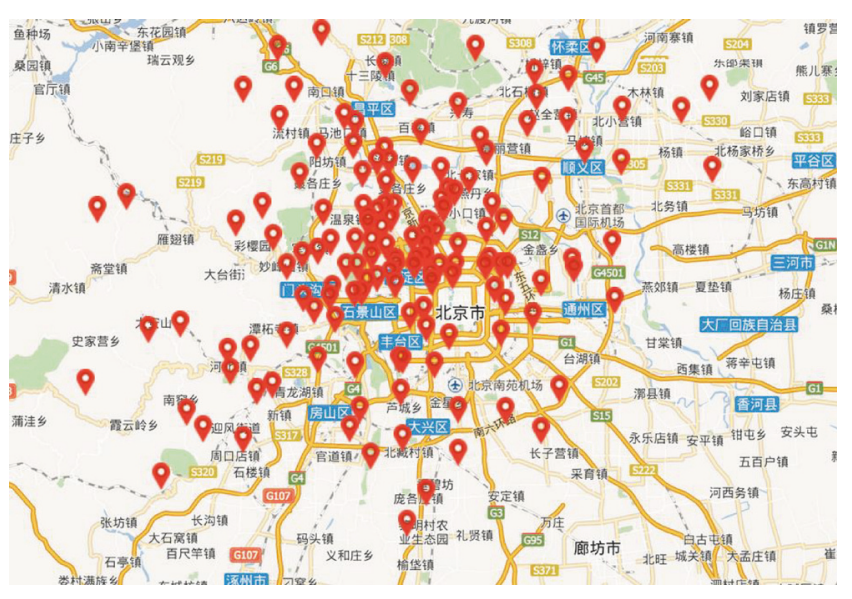

FIGURE 6: Customer nodes.

\section{Conclusions}

In this study, the GOVRP was introduced as a formulation considering the impact of fuel emissions for the thirdparty logistics. The problem was to construct open routes for vehicles to visit all customers with the vehicle capacity constraints. The objective was to minimize the total cost composing of the fuel emissions cost and the driver cost.

A hybrid tabu search algorithm was designed to deal with the GOVRP instances. Experiments were conducted on 60 instances derived from real geographical data of customers in Beijing. We analyzed the cost components with different objectives and compared open routes with closed routes in respects of reducing the total cost. A homogenous fleet was considered in this paper. Computational results showed that vehicle type influenced the total cost and it should be changed 
TABLE 12: Computational results on the 120-node instances by using different vehicles.

\begin{tabular}{|c|c|c|c|c|c|c|}
\hline Type of vehicles & Instance & BS (RMB) & MS (RMB) & WS (RMB) & $\mathrm{SD}$ & ET (s) \\
\hline \multirow{10}{*}{ Only light duty } & BJ120_01 & 21699.63 & 21954.27 & 22209.26 & 135.47 & 15.38 \\
\hline & BJ120_02 & 22803.9 & 23135.32 & 23736.67 & 273.1 & 16.09 \\
\hline & BJ120_03 & 22074.76 & 22569.41 & 22921.39 & 270.03 & 17.46 \\
\hline & BJ120_04 & 22380.9 & 22560.26 & 22764.57 & 138.1 & 16.91 \\
\hline & BJ120_05 & 22602.33 & 23308.71 & 23666.93 & 289.5 & 14.39 \\
\hline & BJ120_06 & 22656.5 & 22975.25 & 23166.4 & 145.56 & 14.44 \\
\hline & BJ120_07 & 21156.14 & 21711.99 & 22170.6 & 301.69 & 14.37 \\
\hline & BJ120_08 & 20837.63 & 21069.17 & 21418.09 & 211.61 & 15.66 \\
\hline & BJ120_09 & 21865.67 & 22195.93 & 22498.88 & 170.26 & 14.43 \\
\hline & BJ120_10 & 22705.98 & 23054.33 & 23556.8 & 217.54 & 14.4 \\
\hline \multirow{10}{*}{ Only medium duty } & BJ120_01 & 23290.23 & 23541.68 & 23691.38 & 124.27 & 12.99 \\
\hline & BJ120_02 & 23133.82 & 23473.76 & 23710.98 & 178.16 & 12.55 \\
\hline & BJ120_03 & 23320.12 & 23573.14 & 23908.95 & 186.7 & 12.9 \\
\hline & BJ120_04 & 23117.27 & 23545.71 & 23757.64 & 194.07 & 13.01 \\
\hline & BJ120_05 & 23687.6 & 24099.96 & 24385.48 & 207.61 & 13.08 \\
\hline & BJ120_06 & 23273.19 & 23621.69 & 23870.13 & 162.68 & 12.49 \\
\hline & BJ120_07 & 23136.08 & 23257.6 & 23424.7 & 91.21 & 13.13 \\
\hline & BJ120_08 & 22752.22 & 23085.88 & 23320.75 & 200.2 & 13.35 \\
\hline & BJ120_09 & 23171.61 & 23279.47 & 23450.24 & 85.55 & 12.65 \\
\hline & BJ120_10 & 23476.59 & 23845.67 & 24258.76 & 222.02 & 12.58 \\
\hline \multirow{10}{*}{ Only heavy duty } & BJ120_01 & 27565.54 & 27974.16 & 28341.07 & 204.51 & 20.49 \\
\hline & BJ120_02 & 27337.33 & 27628.98 & 28138.69 & 243.32 & 18.79 \\
\hline & BJ120_03 & 26855.88 & 27379.48 & 27991.72 & 327.29 & 20.89 \\
\hline & BJ120_04 & 27580.86 & 27834.25 & 28040.53 & 168.47 & 21.69 \\
\hline & BJ120_05 & 28189.69 & 28500.43 & 28755.03 & 186.67 & 18.96 \\
\hline & BJ120_06 & 27679.42 & 27933.79 & 28172.32 & 143.93 & 19.15 \\
\hline & BJ120_07 & 26969.4 & 27668.76 & 28014.39 & 286.72 & 21.03 \\
\hline & BJ120_08 & 26617.8 & 27268.75 & 27742.87 & 315.44 & 22.64 \\
\hline & BJ120_09 & 27000.07 & 27382.75 & 27631.08 & 187.61 & 20.21 \\
\hline & BJ120_10 & 27663.24 & 27887.53 & 28107.04 & 126.69 & 20.54 \\
\hline
\end{tabular}

according to the instance's size. In the future, a heterogeneous fleet of vehicles can be used to minimize the total cost. Other types of the OVRP, such as OVRPTW and MDOVRP, can be solved by our algorithm with some changes.

Nowadays, several novel computing techniques are used to deal with complex problems. Some of them are bioinspired models, such as membrane-inspired evolutionary algorithms [24-26] and probe machine [27]. Their nondeterministic distributed parallel frameworks have been proved to improve the performance of optimization algorithms [28]. Most of them can be used to solve real-life problems [29]. We hope that more competitive results for our GOVRP instances can be obtained by using those algorithms in the future.

\section{Conflicts of Interest}

The authors declare that they have no conflicts of interest.

\section{Acknowledgments}

This work was supported by the National Natural Science Foundation of China (Grant nos. 61502012, 61772290, and
61373066); the Beijing Natural Science Foundation (Grant no. 4164096); the Humanity and Social Science Youth Foundation of Ministry of Education of China (Grant no. 13YJC630010); the Science and Technology Development Strategy Research Program of Tianjin (Grant no. 16ZLZXZF00030); and the Collaborative Innovation Center for China Economy.

\section{References}

[1] L. Schrage, "Formulation and structure of more complex/ realistic routing and scheduling problems," Networks, vol. 11, no. 2, pp. 229-232, 1981.

[2] S. Raff, "Routing and scheduling of vehicles and crews: the state of the art," Computers and Operations Research, vol. 10, no. 2, pp. 63-211, 1983.

[3] J. Brandao, "A tabu search algorithm for the open vehicle routing problem," European Journal of Operational Research, vol. 157, no. 3, pp. 552-564, 2004.

[4] U. Derigs and K. Reuter, "A simple and efficient tabu search heuristic for solving the open vehicle routing problem," Journal of the Operational Research Society, vol. 60, no. 12, pp. 1658-1669, 2009. 
[5] E. E. Zachariadis and C. T. Kiranoudis, "An open vehicle routing problem metaheuristic for examining wide solution neighborhoods," Computers and Operations Research, vol. 37, no. 4, pp. 712-723, 2010.

[6] S. A. Mirhassani and N. Abolghasemi, "A particle swarm optimization algorithm for open vehicle routing problem," Expert Systems with Applications, vol. 38, no. 9, pp. 11547-11551, 2011.

[7] X.-Y. Li, P. Tian, and S. C. H. Leung, "An ant colony optimization metaheuristic hybridized with tabu search for open vehicle routing problems," Journal of the Operational Research Society, vol. 60, no. 7, pp. 1012-1025, 2009.

[8] S. Yu, C. Ding, and K. Zhu, "A hybrid GA-TS algorithm for open vehicle routing optimization of coal mines material," Expert Systems with Applications, vol. 38, no. 8, pp. 10568-10573, 2011.

[9] P. P. Repoussis, C. D. Tarantilis, O. Braysy, and G. Ioannou, "A hybrid evolution strategy for the open vehicle routing problem," Computers and Operations Research, vol. 37, no. 3, pp. 443-455, 2010.

[10] R. Russell, W.-C. Chiang, and D. Zepeda, "Integrating multiproduct production and distribution in newspaper logistics," Computers \& Operations Research, vol. 35, no. 5, pp. 1576-1588, 2008.

[11] C. D. Tarantilis and C. T. Kiranoudis, "Distribution of fresh meat," Journal of Food Engineering, vol. 51, no. 1, pp. 85-91, 2002.

[12] R. Atefi, M. Salari, L. C. Coelho, and J. Renaud, "The open vehicle routing problem with decoupling points," European Journal of Operational Research, vol. 265, no. 1, pp. 316-327, 2018.

[13] C. Erbao, L. Mingyong, and Y. Hongming, "Open vehicle routing problem with demand uncertainty and its robust strategies," Expert Systems with Applications, vol. 41, no. 7, pp. 3569-3575, 2014.

[14] K. Fleszar, I. H. Osman, and K. S. Hindi, "A variable neighbourhood search algorithm for the open vehicle routing problem," European Journal of Operational Research, vol. 195, no. 3, pp. 803-809, 2009.

[15] E. Demir, T. Bektaş, and G. Laporte, "A comparative analysis of several vehicle emission models for road freight transportation," Transportation Research Part D: Transport and Environment, vol. 16, no. 5, pp. 347-357, 2011.

[16] T. Bektaş and G. Laporte, "The pollution-routing problem," Transportation Research Part B: Methodological, vol. 45, no. 8, pp. 1232-1250, 2011.

[17] Ç. Koç, T. Bektaş, O. Jabali, and G. Laporte, "The fleet size and mix pollution-routing problem," Transportation Research Part B: Methodological, vol. 70, pp. 239-254, 2014.

[18] E. Demir, T. Bektaş, and G. Laporte, "A review of recent research on green road freight transportation," European Journal of Operational Research, vol. 237, no. 3, pp. 775-793, 2014.

[19] M. Barth, T. Younglove, and G. Scora, "Development of a Heavy-duty Diesel Modal Emissions and Fuel Consumption Model," Tech. Rep. UCB-ITSPRR-2005-1, California PATH Program, Institute of transportation Studies, University of California at Berkeley, California, Calif, USA, 2005.

[20] M. Scora and G. Barth, "Comprehensive Modal Emission Model (CMEM), Version 3.01, User Guide," Tech. Rep., 2006, http:// www.cert.ucr.edu/cmem/docs/CMEM_User_Guide_v3.01d.pdf.

[21] M. Barth and K. Boriboonsomsin, "Real-world carbon dioxide impacts of traffic congestion," Transportation Research Record, no. 2058, pp. 163-171, 2008.
[22] Man, Trucks in Distribution Transport, http://www.mantruckandbus.co.uk/en/trucks/start_trucks.html.

[23] E. Demir, T. Bektas, and G. Laporte, "An adaptive large neighborhood search heuristic for the Pollution-Routing PROblem," European Journal of Operational Research, vol. 223, no. 2, pp. 346-359, 2012.

[24] G. Zhang, H. Rong, F. Neri, and M. J. Pérez-Jiménez, "An optimization spiking neural $\mathrm{P}$ system for approximately solving combinatorial optimization problems," International Journal of Neural Systems, vol. 24, no. 5, pp. 1-16, 2014.

[25] G. Zhang, H. Rong, J. Cheng, and Y. Qin, "A populationmembrane-system-inspired evolutionary algorithm for distribution network reconfiguration," Chinese Journal of Electronics, vol. 23, no. 3, pp. 437-441, 2014.

[26] X. Zhang, J. Li, and L. Zhang, "A multi-objective membrane algorithm guided by the skin membrane," Natural Computing, vol. 15, no. 4, pp. 597-610, 2016.

[27] J. Xu, "Probe machine," IEEE Transactions on Neural Networks and Learning Systems, vol. 27, no. 7, pp. 1405-1416, 2016.

[28] G. Zhang, J. Cheng, M. Gheorghe, and Q. Meng, "A hybrid approach based on differential evolution and tissue membrane systems for solving constrained manufacturing parameter optimization problems," Applied Soft Computing, vol. 13, no. 3, pp. 1528-1542, 2013.

[29] G. Zhang, M. J. Prez-Jimnez, and M. Gheorghe, Real-Life Applications with Membrane Computing, Springer, Berlin, Germany, 2017. 


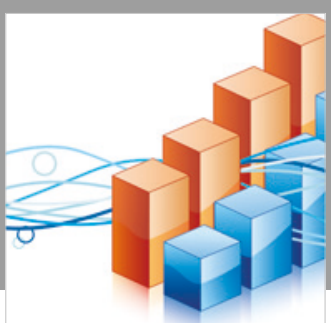

Advances in

Operations Research

\section{-n-m}
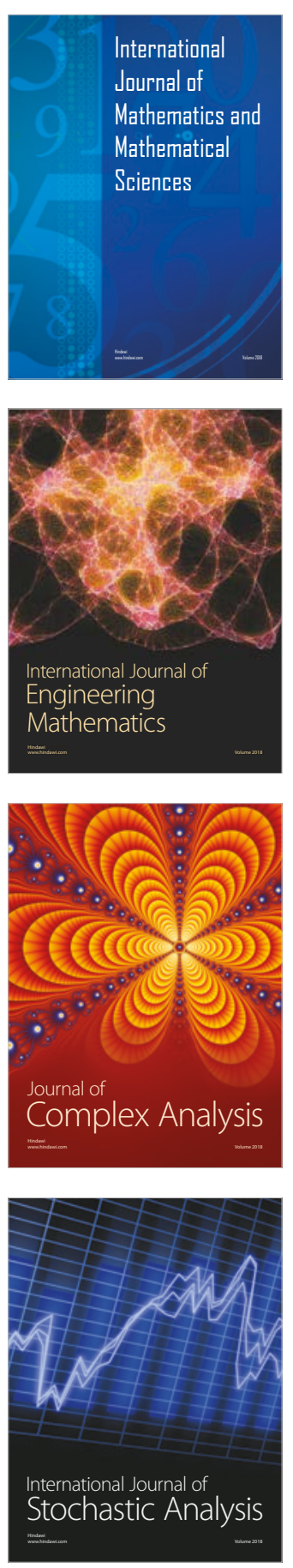
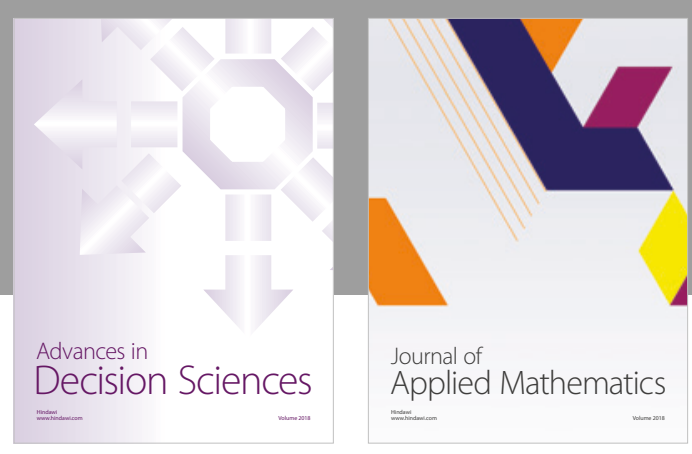

Journal of

Applied Mathematics
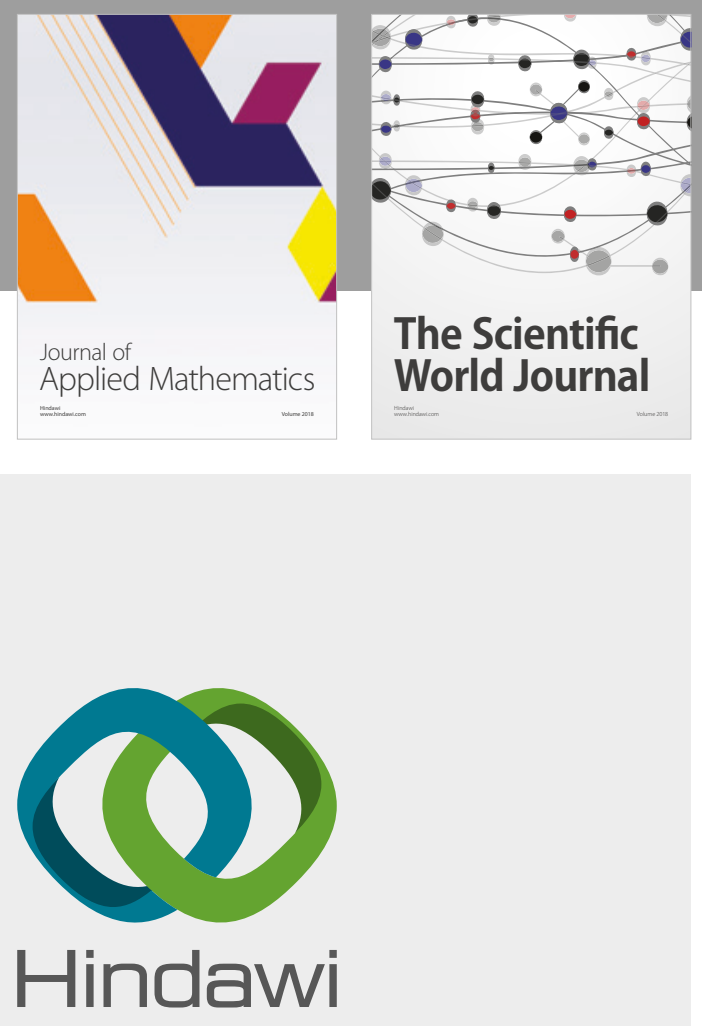

Submit your manuscripts at

www.hindawi.com

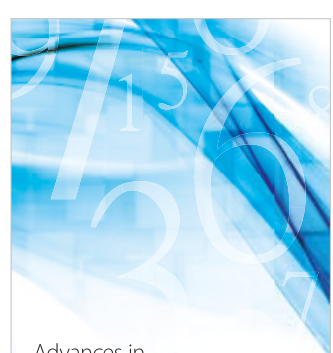

Advances in
Numerical Analysis
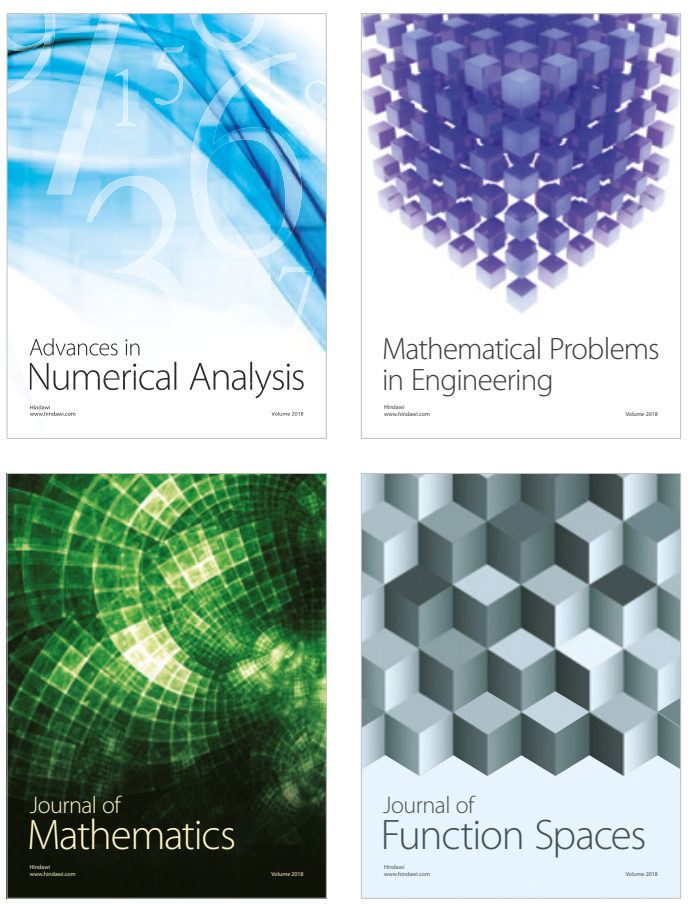

Mathematical Problems in Engineering

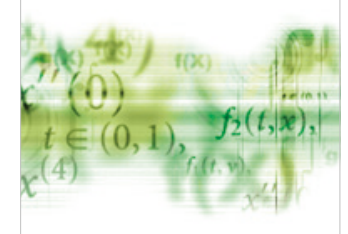

International Journal of

Differential Equations

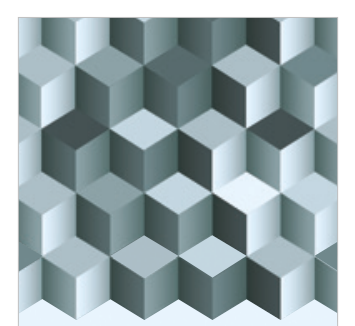

Journal of

Function Spaces
The Scientific

World Journal

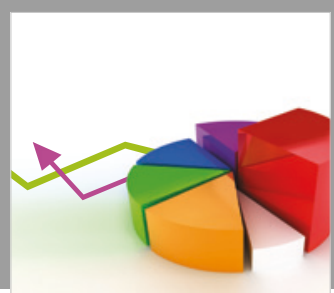

Journal of

Probability and Statistics
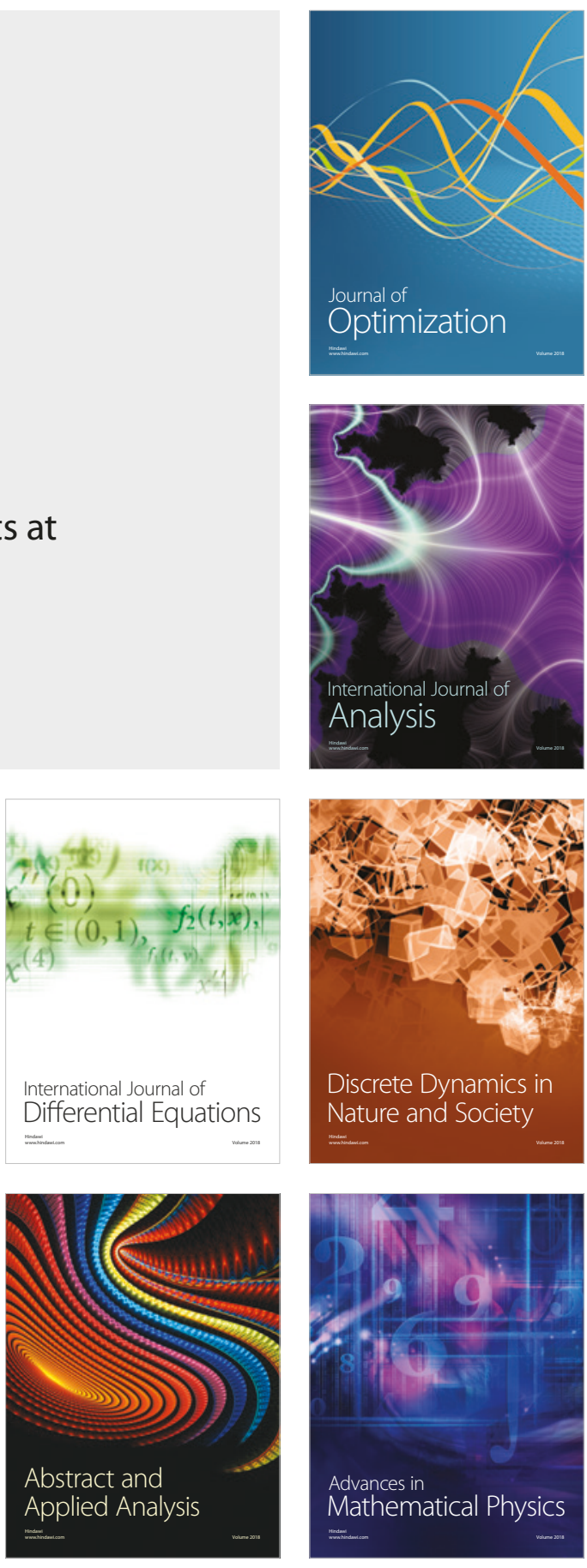